\title{
Free and total sterols in olive oils. Effects of neutralization
}

\author{
By A. Pasqualone and M. Catalano \\ Istituto di Industrie Agrarie, Università di Bari. Via Amendola, 165/A \\ 70126, Bari (Italy).
}

\section{RESUMEN}

\section{Esteroles libres y totales en aceites de oliva. Efectos} de la neutralización

Se ha realizado una determinación directa cuali-cuantitativa por CGL de los esteroles libres y totales, para investigar las variaciones de la relación entre ellos en diferentes categorías de aceite de oliva y estudiar la posibilidad del uso de esta relación como parámetro para la evaluación de la calidad del aceite. Dicha relación se calculó sobre un conjunto de 13 muestras de aceites de oliva virgen y 28 muestras de aceites vírgenes corrientes y lampantes. Para estudiar los efectos de la neutralización sobre los esteroles libres y totales, se determinaron sus contenidos y relación entre ellos tras la neutralización de los aceites vírgenes corrientes y lampantes. También se han analizado los componentes individuales de los esteroles libres y totales, especialmente los más representativos.

El examen total de los valores de la relación esteroles libres $x$ 100 / esteroles totales realizado sobre una población de 41 muestras de aceites naturales y $\mathbf{2 8}$ de aceites neutralizados indicó que, cuando esta relación supera el $70 \%$, puede excluirse la presencia de aceites neutralizados en aceites vírgenes extra.

PALABRAS-CLAVE: Aceite de oliva - Calidad (evaluación) Esteroles - Neutralización (efecto de).

\section{SUMMARY}

Free and total sterols in olive oils. Effects of neutralization

In order to further investigate the variations of the ratio of free to total sterols in different categories of olive oil and to test the possibility to use this ratio as a parameter for olive oil quality evaluation, a direct GLC quali-quantitative determination of either free or total sterols was made, and their ratio calculated, over a set 13 samples of extra virgin oils and over a set of 28 current virgin and lampante oils. In order to study the effects of neutralization on free and total sterols, their amounts and ratio were also determined after the neutralization of the current virgin and lampante oils. The single components of free and total sterols were also analyzed, especially the most represented ones.

The overall examination of the free $\times 100 /$ total sterols' ratio values found over all the samples of 41 natural oils and 28 neutralized oils indicated that, when this ratio exceeds $70 \%$, it can exclude the presence of neutralized oils in extra virgin ones.

KEY-WORDS: Neutralization (effect of) - Olive oil - Quality (evaluation) - Sterols.

\section{INTRODUCTION}

It is now quite accepted by technologists, doctors, nutritionists and, above all, by final consumers, that virgin olive oils represent the best food, within the category of all vegetable and animal alimentary fats.

Actually, within this wide class of oils, there are different chemical and organolectic characteristics and commercial values as related to the different categories of extra virgin, virgin, current virgin and lampante oils. Despite this fact, the EC, Regulations no. $2568 / 91$, no. $183 / 93$ and no. $656 / 95$ have established only differences in such chemical parameters as acidity percentage, so that less valuable oils (current virgin ones or lampante virgin edible ones, for example) could be commercially revalued simply eliminating free fatty acids by neutralization or by distillation at relatively low temperatures (deacidification + deodorization).

The same EC Regulations assign to the different categories of virgin olive oils the corresponding limits for total sterols and for their quali-quantitative composition. However, no indications are provided on the state of sterols themselves, if free or esterified to fatty acids. Actually, all alimentary fats contain both free and esterified sterols, as extensively reported in recent literature.

For example, as far as virgin olive oils are concerned, Kochhar (1983) reports data determined over a single sample and finds a free / esterified sterols' ratio of 2.2. Mariani et al. (1989) analyze, via direct GLC, 19 samples of virgin and lampante oils from a Southern Italy region and find total sterol amounts of $2000-2700 \mathrm{mg} / \mathrm{kg}$ with a higher quantity of stigmasterol within free sterols. Grob et al. (1990) determine total and free campesterol, stigmasterol and $\beta$-sitosterol and find free $\beta$-sitosterol amounts ranging from 71 to $92 \%$ of total $\beta$-sitosterol in virgin olive oils and from 41 to $85 \%$ in lampante oils and in the corresponding rectified ones. Mariani et al. (1991) find much lower amounts of free sterols in rectified olive oils than in virgin ones. Serani and Piacenti (1992), analyzing a set of edible vegetable oils including two raw olive oils and the related rectified ones, observe again a reduction in free sterols after refining. Caponio et al. $(1995,1996)$ analyze, via combined TLC and GLC, current virgin, lampante and the corresponding neutralized olive oils finding, in virgin oils, a ratio of free to esterified sterols greater than 2.0 (with the highest value of 4.3). In the neutralized oils the same ratio lowers below 2 (ranging from 0.9 to 1.8), the decrease of free sterols during neutralization being equal to almost $50 \%$. Mariani and Venturini (1996) determine, via direct GLC, the changes in free / esterified sterols ratio after 18-month storage. They observe a 
significant lowering of free sterol amounts and a subsequent increase in esterified ones.

In the present report, in order to further investigate the variations in the ratio of free to total sterols in different categories of olive oil and to test the possibility to use this ratio as a parameter for olive oil quality evaluation, a direct GLC qualiquantitative determination of both free and total sterols was made, and their ratio calculated, over a set of 13 samples of extra virgin oils and over a set of 28 current virgin and lampante oils. In order to study the effects of neutralization on free and total sterols, their amounts and ratio were also determined after the neutralization of the current virgin and lampante oils. The single components of free and total sterol fractions were also analyzed, especially the most represented ones.

\section{MATERIALS AND METHODS}

Samples. Forty-one samples of surely natural virgin olive oils, directly drawn at the oil-mills, were analyzed. Out of them, 13 belonged to the extra virgin category and 28 to the current virgin and lampante ones.

Acidity percentage determination was made following EC Regulation no. 2568/91, enclosure II (1991).

Neutralization. Samples having acidity percentage values higher than 1.00 were neutralized using the following conditions (similar to that of the discontinuous system in oil industries): oil temperature $40^{\circ} \mathrm{C} ; 5 \%$ $\mathrm{NaOH}$ water solution; repeated washing with water to reach exact neutrality of washing water.

Total sterol determination was made following $C$ 72 N.G.D. (1987) methodology. Oils were first saponified with methanolic $\mathrm{KOH}$, the unsaponifiable fraction was recovered by diethyl ether and the sterol fraction was separated by TLC. Sterols were then transformed in trimethylsilylethers and analyzed via GLC by a $25 \mathrm{~m}$ long, $0.25 \mathrm{~mm}$ internal diameter fused silica capillary column SPB- $5^{\mathrm{TM}}$ (Supelco), film $0.25 \mu$, with $5 \alpha$-cholestan $3 \beta$-ol (Sigma Aldrich) as internal standard.

Free sterols were determined by TLC separation, using $5 \alpha$-cholestan $3 \beta$-ol (Sigma Aldrich) as internal standard, followed by GLC quali-quantitative evaluation. $1.00 \mathrm{~g}$ of oil was supplemented with 0.50 $\mathrm{mg}$ of $5 \alpha$-cholestan $3 \beta$-ol and underwent TLC on $G$ 60 silicagel plates (1.0 $\mathrm{mm}$ thickness) in elution mixture composed of petroleum ether (b.p. $40-60^{\circ} \mathrm{C}$ ): diethyl ether: formic acid (70:30:1.5 v:v:v). The most polar band containing free sterols was recovered and subjected to the same treatments as for total sterols.

\section{RESULTS AND DISCUSSION}

A total number of forty-one samples of surely natural olive oil was analyzed. After the acidity percentage determination, 13 out of the 41 samples resulted to be extra virgin oils, with acidity values below $1 \%$. The remnant 28 samples were found to be current virgin and lampante oils, with acidity values ranging from 2.70 to $11.80 \%$. These 28 oils were neutralized in the laboratory and were analyzed both before and after neutralization.

The data obtained from the analysis of sterol contents in the 13 extra virgin oil samples, reported in Tab. I, showed total sterol contents always greater than $1000 \mathrm{mg} / \mathrm{kg}$ (that is the lowest limit established by current laws) and ranging from 1151 to 1793 $\mathrm{mg} / \mathrm{kg}$ (1418 $\mathrm{mg} / \mathrm{kg}$ on average). In particular, it is evident that free sterols, ranging from 939 to 1394 $\mathrm{mg} / \mathrm{kg}(1126 \mathrm{mg} / \mathrm{kg}$ on average), represented the major component in these oils, with respect to the esterified sterols, leading to a free $\times 100 /$ total sterols'ratio value of $79 \%$ on average and, in any case, greater than $70 \%$ in $93 \%$ of the samples examined.

Total sterols were found to be higher than 1000 $\mathrm{mg} / \mathrm{kg}$ also in the 28 samples of oils belonging to less valuable commercial categories (current virgin and lampante oils) with values varying from 1219 to 2961 $\mathrm{mg} / \mathrm{kg}$ (1901 $\mathrm{mg} / \mathrm{kg}$ on average) (Tab. II). As far as free sterols are concerned, they were the major component of total sterols, with values ranging from $863 \mathrm{up}$ to $2546 \mathrm{mg} / \mathrm{kg}$ (1448 mg/kg on average) and with a free $\times 100 /$ total sterols' ratio comprised between 65.4 and $86.0 \%$ (76. $1 \%$ on average).

In any case, considering the totality of the 41 natural samples, free sterols were always $65 \%$ higher $(77.1 \%$ on average) than total sterols.

These values dropped significantly after the neutralization of current virgin and lampante oils. The neutralized oils (Tab. III), with a very little residual acidity $(0.07-0.29 \%)$ showed, in fact, total sterols varying from 629 to $1331 \mathrm{mg} / \mathrm{kg}$ (mean value 1020 $\mathrm{mg} / \mathrm{kg}$ ). These values were lower than those observed in the same samples before deacidification, with a $52 \%$ loss in total sterols on average. Free sterols showed an even stronger decrease with values ranging, after neutralization, from 353 to $791 \mathrm{mg} / \mathrm{kg}(563 \mathrm{mg} / \mathrm{kg}$ as mean value) and with a $62 \%$ average loss.

The best evaluation of the loss in sterols was expressed by the free $\times 100 /$ total sterols' ratio that was found much lower than before neutralization, with values ranging from 44.8 to $63.1 \%$ and an average value of $55.2 \%$. In particular, while in current virgin and in lampante oils this parameter was always greater than $65 \%$, in the same oils, after neutralization, it was always less or equal to $63 \%$. The ratio was not related to free nor to residual acidity and indicated that the highest losses affected free sterols instead of esterified ones.

The overall examination of the free $\times 100 /$ total sterols' ratio values found over 41 samples of natural oils and 28 neutralized oils revealed that when this 
Table I

Sterol contents in extra virgin oils

\begin{tabular}{ccccc}
\hline $\begin{array}{c}\text { Sample } \\
\text { n. }\end{array}$ & $\begin{array}{c}\text { Acidity } \\
(\%)\end{array}$ & $\begin{array}{c}\text { Total sterols } \\
\text { (mg/kg) }\end{array}$ & $\begin{array}{c}\text { Free sterols } \\
(\mathbf{m g} / \mathbf{k g})\end{array}$ & $\begin{array}{c}\text { Free/total } \\
\text { sterols } \\
(\%)\end{array}$ \\
\hline 1 & 0.92 & 1618 & 1134 & 70.1 \\
2 & 0.85 & 1390 & 939 & 67.6 \\
3 & 0.83 & 1362 & 989 & 72.6 \\
4 & 0.76 & 1226 & 1141 & 93.0 \\
5 & 0.85 & 1151 & 969 & 84.2 \\
6 & 0.75 & 1548 & 1144 & 73.9 \\
7 & 0.81 & 1388 & 1050 & 75.6 \\
8 & 0.78 & 1793 & 1394 & 77.7 \\
9 & 0.92 & 1302 & 1147 & 88.1 \\
10 & 0.70 & 1491 & 1144 & 76.7 \\
11 & 0.61 & 1166 & 1049 & 80.0 \\
12 & 0.43 & 1793 & 1394 & 77.7 \\
13 & 0.58 & 1203 & 1147 & 95.3 \\
\hline mean value & 0.75 & 1418 & 1126 & 79.4 \\
\hline
\end{tabular}

Table II

Sterol contents in current virgin and lampante oils

\begin{tabular}{|c|c|c|c|c|}
\hline $\begin{array}{c}\text { Sample } \\
\text { n. }\end{array}$ & $\begin{array}{l}\text { Acidity } \\
(\%)\end{array}$ & $\begin{array}{c}\text { Total sterols } \\
(\mathrm{mg} / \mathrm{kg})\end{array}$ & $\begin{array}{c}\text { Free sterols } \\
(\mathrm{mg} / \mathrm{kg})\end{array}$ & $\begin{array}{c}\text { Free/total } \\
\text { sterols } \\
(\%)\end{array}$ \\
\hline 1 & 2.70 & 1469 & 1189 & 80.9 \\
\hline 2 & 2.80 & 2961 & 2546 & 86.0 \\
\hline 3 & 4.00 & 2287 & 1607 & 70.3 \\
\hline 4 & 4.00 & 1830 & 1361 & 74.4 \\
\hline 5 & 4.10 & 1712 & 1408 & 82.2 \\
\hline 6 & 4.40 & 1831 & 1199 & 65.4 \\
\hline 7 & 4.50 & 1764 & 1342 & 76.1 \\
\hline 8 & 4.60 & 2039 & 1395 & 68.4 \\
\hline 9 & 4.80 & 1381 & 1027 & 74.4 \\
\hline 10 & 5.00 & 1570 & 1164 & 74.1 \\
\hline 11 & 5.10 & 1272 & 1048 & 82.4 \\
\hline 12 & 5.20 & 1620 & 1279 & 78.9 \\
\hline 13 & 5.30 & 1596 & 1211 & 75.9 \\
\hline 14 & 5.40 & 2277 & 1833 & 80.5 \\
\hline 15 & 5.50 & 1383 & 1150 & 83.1 \\
\hline 16 & 5.70 & 2398 & 1773 & 73.9 \\
\hline 17 & 5.90 & 2198 & 1637 & 74.5 \\
\hline 18 & 6.10 & 2186 & 1710 & 78.2 \\
\hline 19 & 6.20 & 2290 & 1590 & 69.4 \\
\hline 20 & 6.40 & 2292 & 1756 & 76.6 \\
\hline 21 & 6.50 & 2187 & 1835 & 83.9 \\
\hline 22 & 7.60 & 1716 & 1350 & 78.7 \\
\hline 23 & 6.70 & 2409 & 1786 & 74.1 \\
\hline 24 & 7.40 & 1755 & 1261 & 71.8 \\
\hline 25 & 8.30 & 1807 & 1381 & 76.4 \\
\hline 26 & 10.40 & 1912 & 1416 & 74.1 \\
\hline 27 & 11.00 & 1874 & 1423 & 75.9 \\
\hline 28 & 11.80 & 1219 & 863 & 70.8 \\
\hline nean value & 5.98 & 1901 & 1448 & 76.1 \\
\hline
\end{tabular}

Table III

Sterol contents in neutralized oils

\begin{tabular}{|c|c|c|c|c|}
\hline $\begin{array}{c}\text { Sample } \\
\text { n. }\end{array}$ & $\begin{array}{l}\text { Acidity } \\
(\%)\end{array}$ & $\begin{array}{c}\text { Total sterols } \\
(\mathrm{mg} / \mathrm{kg})\end{array}$ & $\begin{array}{l}\text { Free sterols } \\
\text { (mg/kg) }\end{array}$ & $\begin{array}{c}\text { Free/total } \\
\text { sterols } \\
(\%)\end{array}$ \\
\hline 1 & 0.07 & 904 & 508 & 56.2 \\
\hline 2 & 0.09 & 1326 & 791 & 59.6 \\
\hline 3 & 0.14 & 1080 & 650 & 60.2 \\
\hline 4 & 0.26 & 1104 & 610 & 55.2 \\
\hline 5 & 0.25 & 954 & 602 & 63.1 \\
\hline 6 & 0.16 & 894 & 484 & 54.1 \\
\hline 7 & 0.25 & 986 & 584 & 59.2 \\
\hline 8 & 0.16 & 1331 & 700 & 52.6 \\
\hline 9 & 0.26 & 969 & 562 & 58.0 \\
\hline 10 & 0.29 & 909 & 493 & 54.2 \\
\hline 11 & 0.24 & 629 & 353 & 56.1 \\
\hline 12 & 0.26 & 919 & 536 & 58.3 \\
\hline 13 & 0.15 & 843 & 510 & 60.5 \\
\hline 14 & 0.08 & 1100 & 639 & 58.1 \\
\hline 15 & 0.05 & 736 & 436 & 59.2 \\
\hline 16 & 0.17 & 1257 & 671 & 53.4 \\
\hline 17 & 0.25 & 1307 & 734 & 56.2 \\
\hline 18 & 0.15 & 1250 & 743 & 59.4 \\
\hline 19 & 0.08 & 1080 & 549 & 50.8 \\
\hline 20 & 0.25 & 1040 & 496 & 47.7 \\
\hline 21 & 0.16 & 842 & 446 & 53.0 \\
\hline 22 & 0.07 & 1268 & 779 & 61.4 \\
\hline 23 & 0.18 & 1276 & 607 & 47.6 \\
\hline 24 & 0.26 & 965 & 490 & 50.8 \\
\hline 25 & 0.14 & 802 . & 454 & 56.6 \\
\hline 26 & 0.20 & 1035 & 498 & 48.1 \\
\hline 27 & 0.17 & 943 & 473 & 50.1 \\
\hline 28 & 0.21 & 804 & 360 & 44.8 \\
\hline ean value & 0.18 & 1020 & 563 & 55.2 \\
\hline
\end{tabular}

ratio reaches values higher than $70 \%$, it enables to exclude the presence of neutralized oils in extra virgin ones. However, for minor values it might not permit the detection of neutralized oils, especially in virgin ones. In any case, also considering the other analytical data, this parameter could represent an additional tool to express a judgement about the quality of an oil.

Also the composition of total and free sterols, especially the most represented sterols, was determined for extra virgin oil samples (Tab. IV) and for current virgin and lampante oil samples both before (Tab. V) and after neutralization (Tab. VI).

The data obtained in the extra virgin oil samples (Tab. IV) did not show any significant difference in composition between free and total sterol fractions. In current virgin and lampante oils (Tab. V), focussing the attention to campesterol and stigmasterol, we could 
Table IV

Percent composition of total and free sterol fraction in extra virgin olive oils (the most represented sterols)

\begin{tabular}{|c|c|c|c|c|c|c|c|c|c|c|c|c|c|c|c|c|c|c|}
\hline \multirow{2}{*}{$\begin{array}{c}\text { sample } \\
\text { n. }\end{array}$} & \multicolumn{2}{|c|}{ campesterol } & \multicolumn{2}{|c|}{ stigmasterol } & \multicolumn{2}{|c|}{ chlerosterol } & \multicolumn{2}{|c|}{$\begin{array}{c}\beta \text {-sitosterol + } \\
\text { sitostanol }\end{array}$} & \multicolumn{2}{|c|}{$\Delta^{5}$ avenasterol } & \multicolumn{2}{|c|}{$\begin{array}{c}\Delta^{5,24} \text { stigma } \\
\text { stadienol }\end{array}$} & \multicolumn{2}{|c|}{$\begin{array}{c}\Delta^{7} \text { stigma } \\
\text { stenol }\end{array}$} & \multicolumn{2}{|c|}{$\Delta^{7}$ avenasterol } & \multicolumn{2}{|c|}{$\begin{array}{c}\text { appearing } \\
\beta \text { - sitosterol }\end{array}$} \\
\hline & total & free & total & free & total & free & total & free & total & free & total & free & total & free & total & free & total & free \\
\hline 1 & 3.5 & 4.1 & 2.5 & 2.2 & 0.8 & 0.8 & 85.4 & 85.2 & 6.5 & 6.0 & 0.6 & 0.8 & 0.2 & 0.3 & 0.5 & 0.6 & 93.3 & 92.8 \\
\hline 2 & 3.3 & 3.7 & 2.7 & 2.5 & 0.9 & 0.6 & 86.1 & 86.4 & 5.1 & 5.2 & 0.9 & 0.8 & 0.3 & 0.3 & 0.7 & 0.5 & 93.0 & 93.0 \\
\hline 3 & 3.8 & 3.6 & 2.4 & 2.4 & 1.0 & 0.8 & 85.2 & 85.5 & 6.1 & 6.1 & 0.7 & 0.9 & 0.2 & 0.3 & 0.6 & 0.4 & 93.0 & 93.3 \\
\hline 4 & 3.6 & 3.7 & 2.4 & 2.6 & 1.0 & 1.1 & 85.7 & 85.5 & 5.2 & 5.4 & 1.0 & 1.0 & 0.6 & 0.2 & 0.6 & 0.5 & 92.9 & 93.0 \\
\hline 5 & 3.5 & 3.2 & 2.2 & 2.4 & 1.0 & 0.9 & 85.3 & 86.5 & 6.5 & 5.6 & 0.8 & 0.5 & 0.3 & 0.4 & 0.4 & 0.5 & 93.6 & 93.5 \\
\hline 6 & 3.4 & 3.4 & 2.0 & 2.1 & 1.0 & 0.8 & 86.0 & 86.0 & 6.3 & 6.1 & 0.6 & 0.8 & 0.2 & 0.2 & 0.5 & 0.6 & 93.9 & 93.7 \\
\hline 7 & 3.7 & 3.7 & 2.2 & 2.2 & 0.6 & 0.8 & 86.9 & 86.4 & 5.1 & 5.3 & 0.7 & 0.8 & 0.2 & 0.3 & 0.6 & 0.5 & 93.3 & 93.3 \\
\hline 8 & 3.8 & 4.1 & 2.2 & 2.3 & 1.0 & 1.0 & 85.2 & 85.8 & 6.2 & 5.5 & 0.6 & 0.6 & 0.3 & 0.2 & 0.7 & 0.5 & 93.0 & 92.9 \\
\hline 9 & 3.8 & 3.4 & 2.1 & 2.5 & 0.9 & 1.2 & 85.7 & 85.4 & 5.9 & 5.8 & 0.7 & 0.9 & 0.3 & 0.2 & 0.6 & 0.6 & 93.2 & 93.3 \\
\hline 10 & 3.4 & 3.8 & 1.8 & 2.0 & 0.7 & 0.7 & 86.0 & 85.9 & 6.2 & 5.5 & 0.9 & 0.9 & 0.4 & 0.4 & 0.6 & 0.8 & 93.8 & 93.0 \\
\hline 11 & 3.8 & 3.4 & 2.0 & 2.4 & 0.7 & 0.7 & 84.9 & 85.9 & 6.8 & 6.2 & 0.8 & 0.6 & 0.3 & 0.2 & 0.7 & 0.6 & 93.2 & 93.4 \\
\hline 12 & 3.2 & 2.7 & 2.2 & 1.9 & 0.9 & 0.6 & 85.7 & 86.1 & 5.9 & 6.9 & 0.9 & 1.0 & 0.4 & 0.2 & 0.8 & 0.6 & 93.4 & 94.6 \\
\hline 13 & 3.1 & 3.2 & 2.5 & 2.5 & 0.8 & 0.6 & 85.2 & 85.0 & 6.6 & 6.6 & 0.7 & 1.0 & 0.5 & 0.4 & 6.0 & 0.7 & 93.3 & 93.2 \\
\hline $\begin{array}{l}\text { mean } \\
\text { value }\end{array}$ & 3.5 & 3.5 & 2.2 & 2.3 & 0.9 & 0.8 & 85.6 & 85.8 & 6.0 & 5.9 & 0.8 & 0.8 & 0.3 & 0.3 & 1.0 & 0.6 & 93.3 & 93.3 \\
\hline
\end{tabular}

Table V

Percent composition of total and free sterol fraction in current virgin and in lampante olive oils (the most represented sterols)

\begin{tabular}{|c|c|c|c|c|c|c|c|c|c|c|c|c|c|c|c|c|c|c|}
\hline \multirow{2}{*}{$\begin{array}{c}\text { sample } \\
\text { n. }\end{array}$} & \multicolumn{2}{|c|}{ campesterol } & \multicolumn{2}{|c|}{ stigmasterol } & \multicolumn{2}{|c|}{ chlerosterol } & \multicolumn{2}{|c|}{$\begin{array}{c}\beta \text {-sitosterol + } \\
\text { sitostanol }\end{array}$} & \multicolumn{2}{|c|}{$\Delta^{5}$ avenasterol } & \multicolumn{2}{|c|}{$\begin{array}{l}\Delta^{5,24} \text { stigma- } \\
\text { stadienol }\end{array}$} & \multicolumn{2}{|c|}{$\begin{array}{l}\Delta^{7} \text { stigma- } \\
\text { stenol }\end{array}$} & \multicolumn{2}{|c|}{$\Delta^{7}$ avenasterol } & \multicolumn{2}{|c|}{$\begin{array}{c}\text { appearing } \\
\beta \text {-sitosterol }\end{array}$} \\
\hline & total & free & total & free & total & free & total & tree & total & free & total & free & total & free & total & free & total & free \\
\hline 1 & 3.1 & 3.2 & 1.9 & 2.1 & 0.7 & 0.7 & 89.9 & 89.7 & 4.0 & 3.7 & 0.4 & 0.6 & $\operatorname{tr}$ & $\operatorname{tr}$ & tr & $t r$ & 95.0 & 94.7 \\
\hline 2 & 3.5 & 3.6 & 2.2 & 3.1 & 1.0 & 0.9 & 85.2 & 85.6 & 7.0 & 5.9 & 0.5 & 0.4 & 0.2 & 0.1 & 0.4 & 0.4 & 93.7 & 92.8 \\
\hline 3 & 3.7 & 3.4 & 1.6 & 2.2 & 0.7 & 0.8 & 86.5 & 86.8 & 6.3 & 6.3 & 0.5 & 0.4 & 0.1 & $\operatorname{tr}$ & 0.6 & 0.1 & 94.0 & 94.3 \\
\hline 4 & 3.4 & 3.3 & 2.5 & 3.2 & 0.4 & 0.6 & 85.3 & 85.5 & 7.2 & 6.6 & 0.7 & 0.5 & 0.2 & $t r$ & 0.3 & 0.3 & 93.6 & 93.2 \\
\hline 5 & 3.4 & 3.1 & 2.7 & 2.7 & 0.6 & 0.6 & 88.3 & 89.1 & 4.6 & 4.5 & 0.4 & $t r$ & tr & tr & tr & $t r$ & 93.9 & 94.2 \\
\hline 6 & 3.6 & 3.5 & 2.8 & 3.3 & 0.7 & 0.7 & 83.8 & 84.2 & 7.9 & 7.4 & 0.6 & 0.4 & 0.2 & tr & 0.4 & 0.5 & 93.0 & 92.7 \\
\hline 7 & 4.0 & 3.6 & 2.6 & 3.1 & 0.5 & 0.6 & 85.0 & 84.8 & 6.5 & 6.6 & 0.7 & 1.0 & 0.2 & 0.3 & 0.5 & tr & 92.7 & 93.0 \\
\hline 8 & 3.0 & 2.6 & 1.2 & 2.2 & 0.8 & 0.7 & 87.1 & 86.8 & 6.8 & 6.9 & 0.6 & 0.4 & tr & 0.1 & 0.5 & 0.3 & 95.3 & 94.8 \\
\hline 9 & 3.7 & 3.5 & 1.7 & 2.3 & 0.6 & 0.7 & 90.0 & 88.8 & 3.3 & 3.4 & 0.7 & 0.3 & $\operatorname{tr}$ & tr & $\operatorname{tr}$ & $\operatorname{tr}$ & 94.6 & 94.2 \\
\hline 10 & 3.1 & 2.9 & 2.8 & 3.2 & 0.7 & 0.6 & 87.5 & 86.5 & 4.9 & 5.6 & 0.6 & 0.9 & 0.1 & 0.3 & 0.3 & $t r$ & 93.7 & 93.6 \\
\hline 11 & 3.2 & 3.1 & 2.5 & 2.8 & 0.6 & 0.6 & 86.3 & 86.9 & 6.3 & 6.4 & 0.6 & 0.2 & 0.2 & $t r$ & 0.3 & $t r$ & 93.8 & 94.0 \\
\hline 12 & 2.8 & 2.7 & 1.6 & 2.2 & 0.6 & 0.7 & 89.8 & 89.7 & 4.5 & 4.5 & 0.3 & 0.2 & 0.2 & tr & 0.2 & $t r$ & 95.2 & 95.1 \\
\hline 13 & 2.8 & 2.6 & 2.1 & 2.3 & 0.8 & 0.8 & 89.9 & 90.3 & 3.9 & 3.4 & 0.3 & $\operatorname{tr}$ & 0.2 & tr & $\operatorname{tr}$ & $t r$ & 94.9 & 95.1 \\
\hline 14 & 3.5 & 3.4 & 2.8 & 2.5 & 0.9 & 0.7 & 84.4 & 85.2 & 6.8 & 5.8 & 0.8 & 0.7 & 0.5 & 0.4 & 0.3 & 0.3 & 92.9 & 92.4 \\
\hline 15 & 2.8 & 2.9 & 1.2 & 1.2 & 0.6 & 0.6 & 86.4 & 87.2 & 8.4 & 8.1 & 0.6 & $t r$ & tr & $t r$ & $\operatorname{tr}$ & $t r$ & 96.0 & 95.9 \\
\hline 16 & 3.3 & 3.3 & 2.4 & 2.5 & 0.7 & 0.9 & 85.3 & 85.2 & 6.6 & 6.7 & 0.8 & 0.7 & 0.4 & 0.3 & 0.5 & 0.4 & 93.4 & 93.5 \\
\hline 17 & 3.6 & 3.2 & 2.6 & 2.4 & 0.8 & 0.7 & 84.3 & 85.4 & 7.5 & 6.8 & 0.5 & 0.6 & 0.2 & 0.5 & 0.5 & 0.2 & 93.1 & 93.5 \\
\hline 18 & 3.6 & 3.3 & 2.3 & 2.8 & 0.6 & 0.5 & 86.6 & 89.9 & 6.5 & 3.5 & 0.4 & $t r$ & tr & tr & tr & tr & 94.1 & 93.9 \\
\hline 19 & 2.9 & 2.1 & 2.5 & 3.2 & 0.8 & 0.8 & 85.0 & 86.6 & 7.4 & 5.4 & 0.7 & 0.9 & 0.2 & $\operatorname{tr}$ & 0.5 & tr & 93.9 & 93.7 \\
\hline 20 & 3.4 & 3.4 & 2.4 & 3.3 & 0.9 & 1.0 & 85.2 & 85.0 & 6.7 & 6.7 & 0.6 & 0.4 & 0.2 & 0.1 & 0.6 & 0.1 & 93.4 & 93.1 \\
\hline 21 & 3.3 & 3.1 & 1.5 & 1.6 & 0.8 & 0.6 & 89.1 & 88.8 & 4.8 & 5.5 & 0.5 & 0.2 & tr & 0.2 & tr & $t r$ & 95.2 & 95.1 \\
\hline 22 & 3.5 & 3.6 & 2.1 & 2.2 & 0.7 & 0.7 & 88.3 & 88.2 & 5.4 & 5.3 & $\operatorname{tr}$ & tr & tr & $t r$ & tr & tr & 94.4 & 94.2 \\
\hline 23 & 3.8 & 3.8 & 2.2 & 2.9 & 0.6 & 0.6 & 85.4 & 85.7 & 6.6 & 6.5 & 0.7 & tr & 0.2 & tr & 0.5 & 0.5 & 93.3 & 92.8 \\
\hline 24 & 3.5 & 3.6 & 1.8 & 2.3 & 0.5 & 0.6 & 88.2 & 88.4 & 5.4 & 4.8 & 0.4 & 0.3 & 0.1 & $t r$ & 0.1 & $t r$ & 94.5 & 94.1 \\
\hline 25 & 3.2 & 3.3 & 1.8 & 2.2 & 0.6 & 0.7 & 88.8 & 88.6 & 4.7 & 4.8 & 0.6 & 0.2 & 0.1 & 0.2 & 0.2 & $t r$ & 94.7 & 94.3 \\
\hline 26 & 3.3 & 3.0 & 1.9 & 2.3 & 0.5 & 0.6 & 88.9 & 89.5 & 4.2 & 3.9 & 0.6 & 0.5 & 0.5 & 0.2 & 0.1 & $t r$ & 94.2 & 94.5 \\
\hline 27 & 3.4 & 3.4 & 3.1 & 3.4 & 0.6 & 0.7 & 85.2 & 85.5 & 6.7 & 6.8 & 0.5 & $t r$ & 0.3 & 0.2 & 0.2 & $t r$ & 93.0 & 93.0 \\
\hline 28 & 3.2 & 2.9 & 3.1 & 2.3 & 0.5 & 0.5 & 83.1 & 83.7 & 9.1 & 9.6 & 0.7 & 0.2 & 0.3 & 0.4 & $\operatorname{tr}$ & 0.4 & 93.4 & 94.0 \\
\hline $\begin{array}{l}\text { mean } \\
\text { value }\end{array}$ & 3.3 & 3.2 & 2.2 & 2.6 & 0.7 & 0.7 & 86.7 & 87.1 & 6.1 & 5.8 & 0.6 & 0.5 & 0.2 & 0.3 & 0.4 & 0.3 & 94.0 & 93.9 \\
\hline
\end{tabular}


Table VI

Percent composition of total and free sterol fraction in neutralized olive oils (the most represented sterols)

\begin{tabular}{|c|c|c|c|c|c|c|c|c|c|c|c|c|c|c|c|c|c|c|}
\hline \multirow{2}{*}{$\begin{array}{c}\text { sample } \\
\mathrm{n} .\end{array}$} & \multicolumn{2}{|c|}{ campesterol } & \multicolumn{2}{|c|}{ stigmasterol } & \multicolumn{2}{|c|}{ chlerosterol } & \multicolumn{2}{|c|}{$\begin{array}{c}\beta \text {-sitosterol + } \\
\text { sitostanol }\end{array}$} & \multicolumn{2}{|c|}{$\Delta^{5}$ avenasterol } & \multicolumn{2}{|c|}{$\begin{array}{l}\Delta^{5,24} \text { stigma- } \\
\text { stadienol }\end{array}$} & \multicolumn{2}{|c|}{$\begin{array}{l}\Delta^{7} \text { stigma- } \\
\text { stenol }\end{array}$} & \multicolumn{2}{|c|}{$\Delta^{7}$ avenasterol } & \multicolumn{2}{|c|}{$\begin{array}{c}\text { appearing } \\
\beta \text { - sitosterol }\end{array}$} \\
\hline & total & free & total & free & total & free & total & free & total & free & total & free & total & free & total & free & total & free \\
\hline 1 & 3.2 & 2.9 & 1.6 & 2.1 & 0.7 & 0.7 & 88.6 & 89.9 & 4.6 & 4.0 & 0.7 & 0.4 & 0.2 & $\operatorname{tr}$ & 0.4 & tr & 94.6 & 95.0 \\
\hline 2 & 2.9 & 3.0 & 1.9 & 2.2 & 0.8 & 1.1 & 85.7 & 85.6 & 7.0 & 7.2 & 0.6 & 0.5 & 0.3 & 0.1 & 0.8 & 0.3 & 94.1 & 94.4 \\
\hline 3 & 3.4 & 3.2 & 1.1 & 2.8 & 0.7 & 0.8 & 87.2 & 86.1 & 6.9 & 6.4 & 0.5 & 0.6 & 0.2 & tr & tr & 0.1 & 95.3 & 93.9 \\
\hline 4 & 3.9 & 3.9 & 2.4 & 3.1 & 0.4 & 0.2 & 86.3 & 86.9 & 5.1 & 5.9 & 1.2 & tr & 0.3 & $t r$ & 0.4 & tr & 93.0 & 93.0 \\
\hline 5 & 3.4 & 3.3 & 1.7 & 2.2 & 0.7 & 0.5 & 87.9 & 88.1 & 5.1 & 5.1 & 0.6 & 0.6 & 0.2 & 0.2 & 0.4 & $t r$ & 94.3 & 94.3 \\
\hline 6 & 3.8 & 4.0 & 2.7 & 3.0 & 0.4 & 0.4 & 86.2 & 85.3 & 6.2 & 7.3 & 0.7 & tr & $\operatorname{tr}$ & tr & $\operatorname{tr}$ & tr & 93.5 & 93.0 \\
\hline 7 & 4.0 & 4.2 & 2.7 & 2.3 & 0.4 & 0.6 & 89.0 & 87.3 & 3.8 & 5.5 & tr & tr & $\operatorname{tr}$ & 0.1 & $\operatorname{tr}$ & tr & 93.2 & 93.4 \\
\hline 8 & 3.7 & 3.4 & 2.0 & 1.8 & 0.8 & 0.8 & 85.8 & 86.1 & 6.7 & 7.3 & 0.7 & 0.6 & 0.2 & tr & $\operatorname{tr}$ & tr & 94.1 & 94.8 \\
\hline 9 & 3.5 & 3.4 & 2.5 & 2.2 & 0.8 & 0.7 & 88.6 & 89.4 & 4.1 & 3.5 & 0.5 & 0.6 & $\operatorname{tr}$ & 0.1 & tr & 0.1 & 94.0 & 94.2 \\
\hline 10 & 3.1 & 3.7 & 2.2 & 2.3 & 0.7 & 0.6 & 87.3 & 87.6 & 5.4 & 5.1 & 0.7 & 0.7 & 0.2 & tr & 0.4 & tr & 94.1 & 94.0 \\
\hline 11 & 3.2 & 2.8 & 2.2 & 2.3 & 0.7 & 1.9 & 86.7 & 86.2 & 6.1 & 6.2 & 0.5 & 0.6 & 0.3 & tr & 0.3 & tr & 94.0 & 94.9 \\
\hline 12 & 2.6 & 2.6 & 1.4 & 1.8 & 0.8 & 0.8 & 89.3 & 89.4 & 5.1 & 5.8 & 0.4 & 0.6 & $\operatorname{tr}$ & tr & 0.4 & tr & 95.6 & 95.6 \\
\hline 13 & 2.9 & 3.1 & 1.8 & 2.5 & 0.7 & 0.6 & 88.9 & 87.9 & 4.5 & 4.8 & 0.6 & 0.6 & 0.2 & 0.5 & 0.4 & tr & 94.7 & 93.9 \\
\hline 14 & 3.5 & 3.2 & 2.0 & 2.4 & 0.7 & 1.0 & 85.7 & 86.1 & 6.5 & 6.7 & 0.7 & 0.3 & 0.2 & 0.1 & 0.7 & 0.2 & 93.6 & 94.1 \\
\hline 15 & 3.2 & 3.4 & 1.2 & 1.4 & 0.6 & 0.7 & 86.5 & 87.1 & 7.7 & 7.4 & 0.6 & $t r$ & 0.2 & tr & $\operatorname{tr}$ & tr & 95.4 & 95.2 \\
\hline 16 & 3.7 & 3.6 & 2.4 & 2.5 & 0.9 & 0.6 & 85.6 & 84.7 & 6.2 & 7.2 & 0.8 & 1.0 & tr & tr & 0.4 & 0.4 & 93.5 & 93.5 \\
\hline 17 & 3.5 & 3.5 & 3.0 & 2.6 & 1.0 & 1.0 & 84.3 & 84.5 & 6.9 & 7.0 & 0.6 & 0.4 & $\operatorname{tr}$ & 0.2 & 0.7 & 0.8 & 92.8 & 92.9 \\
\hline 18 & 3.5 & 3.3 & 2.0 & 2.9 & 0.6 & 0.8 & 86.9 & 87.2 & 6.2 & 5.8 & 0.5 & $t r$ & 0.3 & $t r$ & $\operatorname{tr}$ & tr & 94.2 & 93.8 \\
\hline 19 & 3.2 & 2.8 & 2.7 & 2.0 & 0.9 & 0.9 & 84.9 & 85.7 & 6.9 & 6.9 & 0.6 & 0.8 & 0.3 & 0.3 & 0.5 & 0.6 & 93.3 & 94.3 \\
\hline 20 & 3.0 & 2.8 & 2.7 & 2.7 & 1.0 & 0.9 & 85.3 & 86.1 & 6.6 & 6.5 & 0.5 & 0.4 & 0.3 & 0.2 & 0.6 & 0.4 & 93.4 & 93.9 \\
\hline 21 & 3.4 & 3.1 & 1.5 & 1.7 & 0.7 & 0.7 & 89.1 & 89.3 & 4.6 & 4.9 & 0.4 & 0.3 & $\operatorname{tr}$ & tr & 03 & tr & 94.8 & 95.2 \\
\hline 22 & 3.2 & 2.9 & 2.4 & 2.8 & 0.6 & 0.7 & 89.5 & 88.4 & 4.2 & 4.4 & $\mathrm{tr}$ & 0.8 & 0.1 & tr & $\operatorname{tr}$ & tr & 94.3 & 94.3 \\
\hline 23 & 3.6 & 3.7 & 1.6 & 3.0 & 0.4 & 0.4 & 87.7 & 89.2 & 5.2 & 3.7 & 0.9 & $t r$ & 0.2 & tr & 0.4 & tr & 94.2 & 93.3 \\
\hline 24 & 3.7 & 3.4 & 1.6 & 2.4 & 0.6 & 0.8 & 89.4 & 89.2 & 3.7 & 3.7 & 0.5 & 0.5 & 0.3 & tr & 0.2 & $t r$ & 94.2 & 94.2 \\
\hline 25 & 3.3 & 2.7 & 1.8 & 2.4 & 0.7 & 1.0 & 89.2 & 88.9 & 4.4 & 4.0 & 0.4 & 0.5 & $\operatorname{tr}$ & 0.5 & 0.2 & tr & 94.7 & 94.4 \\
\hline 26 & 3.3 & 2.9 & 1.7 & 2.5 & 0.7 & 0.8 & 89.4 & 88.7 & 4.1 & 4.3 & 0.5 & 0.8 & 0.3 & $t r$ & $\operatorname{tr}$ & $t r$ & 94.7 & 94.6 \\
\hline 27 & 3.3 & 3.1 & 2.8 & 2.5 & 0.4 & 0.5 & 86.6 & 87.0 & 5.6 & 5.5 & 0.6 & 0.9 & 0.3 & 0.2 & 0.4 & 0.3 & 93.2 & 93.9 \\
\hline 28 & 3.3 & 3.2 & 2.5 & 2.9 & 0.3 & 0.4 & 84.7 & 85.6 & 7.8 & 6.4 & 0.6 & 1.1 & 0.3 & tr & 0.5 & 0.4 & 93.4 & 93.5 \\
\hline $\begin{array}{l}\text { mean } \\
\text { value }\end{array}$ & 3.4 & 3.3 & 2.1 & 2.4 & 0.7 & 0.7 & 87.2 & 87.3 & 5.6 & 5.7 & 0.6 & 0.6 & 0.2 & 0.2 & 0.4 & 0.4 & 94.1 & 94.1 \\
\hline
\end{tabular}

only find very slight differences in the composition of free sterols as compared to total ones. Campesterol was comprised between 2.8 and $4.0 \%$, with an average of $3.34 \%$, into the total sterol fraction and ranged from 2.1 to $3.8 \%$, with an average of $3.19 \%$, into the free sterol fraction; stigmasterol ranged from 1.2 to $3.1 \%(2.2 \%$ on average $)$ in total fraction and from 1.2 to $3.4 \%(2.56 \%$ on average) in free fraction.
Sterol compositions of current virgin and lampante oils, after neutralization, are reported in Tab. VI. As for campesterol and stigmasterol, slight differences in composition between free and total fractions were observed. Total campesterol ranged from 2.6 to $4.0 \%$ (3.4\% on average) and free campesterol from 2.7 to $4.2 \%$ ( $3.3 \%$ on average). Total stigmasterol ranged from 1.2 to $3.0 \%$ (2.07\% on average) and 
free stigmasterol from 1.4 to $3.1 \%(2.40 \%$ on average).

Thus both free and total campesterol and stigmasterol did not show any significant variations after neutralization, hence indicating that this process affects indistinctly these kinds of sterols.

Some differences were found in $\Delta^{7}$-avenasterol and in $\Delta^{5,24}$-stigmastadienol amounts which resulted lower in current virgin, lampante and neutralized oils than in extra virgin ones. Infact, $\Delta^{7}$-avenasterol showed mean values of $0.3 \%$ (free) and $0.1 \%$ (total) both in current virgin and in lampante and neutralized oils while they ranged from $1.0 \%$ (free) to $0.6 \%$ (total) in extra virgin oils; $\Delta^{5,24}$-stigmastadienol showed mean values of 0.5 and $0.4 \%$ (free and total, respectively) in current virgin and lampante oils, 0.6 and $0.5 \%$ (free and total) in neutralized oils and $0.8 \%$ both for free and for total fraction in extra virgin oils.

\section{CONCLUSIONS}

Over all the 41 samples belonging to the different commercial categories of extra virgin, current virgin and lampante oils, the ratio free $\times 100 /$ total sterols was shown to be greater than $65 \%$. In extra virgin oils the same ratio was above $70 \%$ in $93 \%$ of the samples being analyzed.

The neutralized oils showed a $50 \%$ loss in total sterols and $60 \%$ loss in free sterols. As a consequence, in these oils the ratio free $\times 100 /$ total sterols was always comprised between 44.8 and $63.1 \%$.

Based on these results, this ratio could represent a further tool for the evaluation of extra virgin oil quality and somehow a proof of their genuinity. It would be thus desirable to determine the free $\times 100 /$ total sterols' ratio as a quality index for extra virgin oils able to exclude, for values over $70 \%$, the presence of neutralized oils. This is a quite interesting result because, despite the provisions included in the EC Regulation no. 2568/91 and in the following ones, it could still be possible to assign the extra virgin class to both virgin, current virgin and lampante oils if previously neutralized, deodorized and appropriately mixed with extra virgin ones.
No differences, however, were detected in the composition of free sterol fraction with respect to the total one, except for a slightly lower percentage of free campesterol balanced by slightly higher stigmasterol values both in current virgin and in lampante and neutralized oils. No differences in sterol fraction composition were found between virgin and neutralized oils.

\section{REFERENCES}

Caponio, F., Catalano, M., De Leonardis, T., Comes, S. (1995). Steroli liberi e steroli esterificati negli oli d'oliva. Atti $2^{\circ}$ Congr. It. Scienza e Tecnologia degli Alimenti, Cernobbio, 323-329.

Caponio, F., De Leonardis, T., Catalano, M. (1996). Influenza della neutralizzazione sulla evoluzione degli steroli liberi ed esterificati negli oli d'oliva. Atti XVII Congr. Naz. Merceologia, Lecce, I, 655-660.

(E.C.) (1991). Commission Regulation no. 2568/91, $07 / 11 / 91$, on the characteristics of olive oil and residue oil and on the relevant methods of analysis. E. C. Official Journal, L248, 09/05/91.

Grob, K., Lanfranchi, M., Mariani, C. (1990). Evaluation of olive oils through the fatty alcohols, the sterols and their esters by coupled LC-GC. J. Am. Oil Chem. Soc., $67,10,626-634$.

Kochhar, S.P. (1983). Influence of processing on sterols of edible vegetable oils. Prog. Lipid Res., 22,161-188.

Mariani, C., Fedeli, E., Bovio, V. (1989) Individuazione di oli di estrazione in quelli di pressione. Nota 2. Lampanteanti pugliesi. Riv. It. Sost. Grasse, LXVI, 7, 447-452.

Mariani, C., Venturini, S. (1996). Sull'aumento delle cere durante la conservazione degli oli di oliva. Riv. It. Sost. Grasse, LXXIII, 11, 489-497.

Mariani, C., Fedeli, E., Grob, K. (1991). Valutazione dei componenti minori liberi ed esterificati nelle sostanze grasse. Riv. It. Sost. Grasse, LXVIII, 5, 233-242.

(N.G.D.) (1987) Norme Italiane per il controllo dei grassi e derivati. Staz. Sper. Ind. Oil e Grassi, Milano.

Serani, A., Piacenti, D. (1992). I fenomeni chimico-fisici che regolano la perdita degli steroli liberi durante la raffinazione degli oli vegetali. Riv. It. Sost. Grasse, LXIX, 6, 311-315. 